\title{
Mutaciones genéticas que confieren resistencia a Isoniacida en pacientes con tuberculosis en Lima Centro, Perú 2017-2018.
}

\section{Genetic mutations that confer resistance to Isoniazid in patients with Tuberculosis in Lima Centro, Perú 2017- 2018.}

Correspondencia Kenneth Grenis Vargas Ponce grenisvp@gmail.com

Recibido: 24/10/2020 Aprobado: 05/07/2021

Citar como: Vargas-Ponce KG, Trujillo-Valencia CL, Saravia-Ruiz T, SalasLópez JA, Llanos-Tejada FK. Mutaciones genéticas que confieren resistencia a Isoniacida en pacientes con Tuberculosis en Lima Centro, Perú 2017-2018. Acta Med Peru. 2021:38(2):151-3. doi: https://doi.org/10.35663/ amp.2021.382.1892
Kenneth G. Vargas-Ponce 1,a, Claudia L. Trujillo-Valencia 2,b , Trilce Saravia-Ruiz 2,b, Juan A. Salas-López ${ }^{1,3, c}$, Félix K. Llanos-Tejada 4,5,c

Servicio de Neumología Hospital Nacional Dos De Mayo. Lima, Perú

Universidad San Martin de Porres

Facultad de Medicina Humana, Universidad Nacional Mayor de San Marcos

Instituto de Investigación en Ciencias Biomédicas (INICIB), Universidad Ricardo Palma

Facultad de Medicina Humana, Universidad Ricardo Palma

Médico residente de Neumología

Alumno de la Facultad de Medicina

Médico Neumólogo

\section{RESUMEN}

Se reporta la frecuencia de mutaciones genéticas KatG e inhA que confieren resistencia a isoniacida en una muestra de 777 pacientes con resistencia a isoniacida. Se utilizó la prueba GenoType ${ }^{\circledR}$ MTBDRplus y la prueba de sensibilidad convencional por el método de agar en placa. Se encontró que 54 \% presentó mutación en el gen KatG; este se asoció con resistencia a estreptomicina $76,6 \%(p<0.05)$, rifampicina $66.7 \%(p<0.05)$ y etionamida en un $33 \%(p<0.05)$. La mutación en el gen inhA tuvo una frecuencia de $46 \%$ y se asoció con resistencia a etionamida en un $68,1 \%(p<0.05)$, rifampicina $47,2 \%(p<0,05)$ y estreptomicina $33 \%(p<0,05)$. En estos pacientes, la presencia de genes que confieren resistencia a isoniazida se relacionó con resistencia a otros medicamentos antituberculosos.

Palabras claves: Mycobacterium tuberculosis; Tuberculosis resistente a múltiples medicamentos; Mutación; Isoniacida; Perú.

\section{ABSTRACT}

This a report of the frequency of KatG and inhA genetic mutations that confer resistance to isoniazid in a sample of 777 patients with resistance to isoniazid. GenoType ${ }^{\circledR}$ MTBDRplus test and conventional sensitivity tests by the agar plate method were used. It was found that $54 \%$ presented mutation in the KatG gene, associated with higher resistance to streptomycin $76.6 \%$ ( $p$ $<0.05)$, rifampicin $66.7 \%(p<0.05)$ and ethionamide in $33 \%(p<0.05)$. inhA gene mutation has a frequency of $46 \%$ and was associated with resistance to ethionamide in $68.1 \%(p<0.05)$, rifampicin $47.2 \%(p<0.05)$ and streptomycin 33\% ( $<<0.05)$. In this sample, the presences of mutations that confer resistance to isoniazid was associated with resistance to other antituberculosis drugs.

Keywords: Mycobacterium tuberculosis; Tuberculosis, Multidrug-Resistant; Mutation; Isoniazid; Peru. 
Sr Editor:

La tuberculosis (TB) es una enfermedad prevenible y curable. La Organización Mundial de la Salud (OMS) reporta que alrededor de 10 millones de personas se enfermaron de TB con 1,2 millones de muertes el 2018, siendo la principal causa de muerte infecciosa del mundo. En Perú se reportaron 42940 casos de TB el año 2019, ocupando el décimo primer lugar de las causas de muerte en general ${ }^{[1,2]}$, la resistencia a los antimicrobianos representa una gran amenaza para la salud y la seguridad mundial, se estima que el $3.3 \%$ de los pacientes con TB recién diagnosticada y el $20.1 \%$ de aquellos con TB previamente tratada tienen resistencia a rifampicina $(R)$ e isoniacida $(H)$, lo que se denomina multidrogoresistente (MDR), en Perú los últimos 2 años se han reportado más de 1500 pacientes TB-MDR por año, siendo Lima Metropolitana y el Callao los que notificaron un $70 \%$ de los $\operatorname{casos}^{[3]}$.

La rifampicina e isoniacida representan la piedra angular de tratamiento de primera, la $\mathrm{H}$ es fundamental no solo para el tratamiento de la TB activa, sino también para la terapia preventiva ${ }^{[4]}$. Las personas infectadas con una cepa de TB que es resistente a los medicamentos utilizados para el tratamiento, especialmente la $\mathrm{R}$ y/o la $\mathrm{H}$, tienen una menor probabilidad de curarse y un mayor riesgo de muerte ${ }^{[4]}$, por ello es muy importante la vigilancia de la resistencia a los medicamentos antituberculosos con pruebas de rutina a todos los pacientes con TB y así tener un mejor control de la tuberculosis, pudiendo tener regímenes de tratamiento apropiados y oportunos ${ }^{[5]}$. Existen métodos convencionales para la detección de la susceptibilidad a los fármacos, los que suelen tomar tiempo y elaboración, por eso es necesario el uso de métodos más rápidos, como el uso de sondas de línea, entre los cuales se cuenta la prueba comercial Genotype ${ }^{\circledR}$ MTBDRplus (Hain Lifescience $\mathrm{GmbH}$, Nehren, Germany), con sensibilidad de $97 \%$ y especificidad de $99 \%$ para $\mathrm{R}$, y de $90 \%$ y $99 \%$ para $\mathrm{H}$, respectivamente, en muestras pulmonares con baciloscopia positiva, esta prueba detecta mutaciones en el gen rpoB asociadas con resistencia a la rifampicina, así como resistencia a isoniacida de alto nivel debido a mutaciones en el gen katG y resistencia de bajo nivel debido a mutaciones del gen inhA ${ }^{[6]}$.

El objetivo del presente estudio fue establecer la frecuencia de mutaciones asociadas con la resistencia a la isoniacida mediante la técnica GenoType MTBDRplus en la DIRIS Lima Centro durante el periodo 2017-2018, asimismo, se discute las implicaciones de nuestros hallazgos con la finalidad de lograr un mejor diagnóstico y tratamiento. Para esto se realizó un estudio observacional descriptivo transversal, se evaluaron las fichas de los expedientes aprobados en el Comité de Evaluación de Retratamiento (CER) para casos de tuberculosis resistente de la DIRIS Lima Centro, para resistencia se utilizó la prueba molecular de GenoType ${ }^{\circledR}$ MTBDRplus y la prueba de sensibilidad convencional por el método de agar en placa, se procedió a la tabulación de los datos durante el periodo 2017-2018 los datos fueron digitados y procesados en Microsoft Excel 2016. Se realizó un análisis estadístico descriptivo y analítico, mediante medidas de tendencia central, distribución de frecuencias, chi cuadrado para variables cualitativas.
Tabla 1. Prevalencia del Gen inh A detectada mediante Genotype MTBDRplus, Lima Centro, Perú 2017-2018 N=357(46\% de 777 casos)

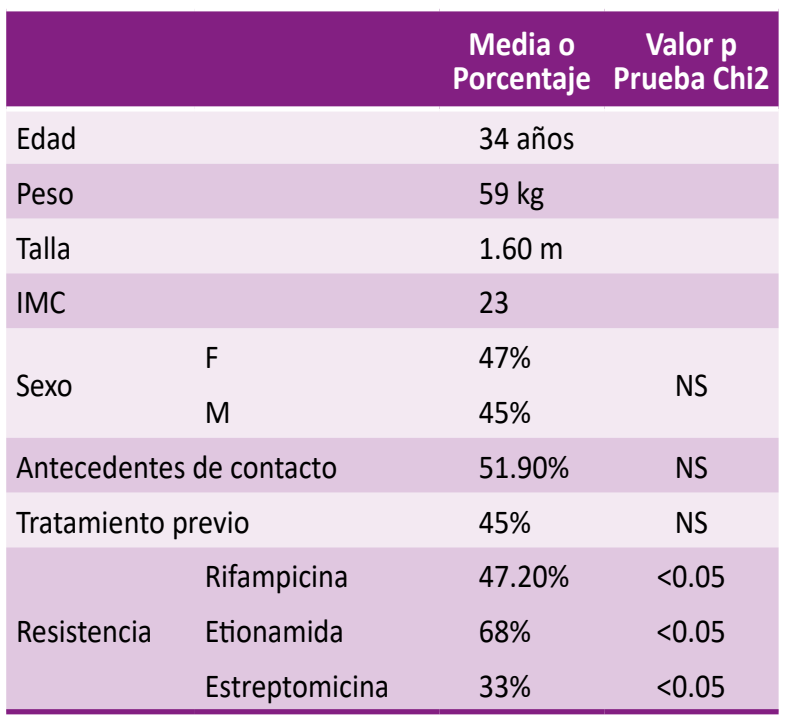

Se identificaron 777 pacientes resistentes a isoniacida, más de la mitad de los pacientes fue de sexo masculino $68 \%$, la edad promedio fue de 34 años con un rango de edad entre 17 a 72 años, el $72.2 \%$ de los pacientes no recibió tratamiento antituberculoso previo $(\mathrm{p}<0.05)$, la mutación más frecuente fue la del gen katG en un $54 \%$, similar con investigaciones previas que identificaron a katG como el gen predominante asociado con la resistencia a isoniacida ${ }^{[4,6,]}$, seguido de el gen inhA en un $46 \%$, las mutaciones en el gen katG se asociaron con la resistencia a estreptomicina $76,7 \%(p<0.05)$ seguido de rifampicina en un $66,7 \%$ $(p<0.05)$ y en menor porcentaje a etionamida $(33 \%)(p<0.05)$, las mutaciones en el gen inhA se asociaron con la resistencia a

Tabla 2. Prevalencia del Gen kat $\mathrm{G}$ detectada mediante Genotype MTBDRplus, Lima Centro, Perú 2017-2018 N= 420(54\% de 777 casos)

\begin{tabular}{llll} 
& & \multicolumn{1}{c}{$\begin{array}{c}\text { Media o } \\
\text { Porcentaje }\end{array}$} & $\begin{array}{c}\text { Valor } \mathbf{p} \\
\text { Prueba Chi2 }\end{array}$ \\
\hline Edad & & 34 años & \\
\hline Peso & & $58 \mathrm{~kg}$ & \\
\hline Talla & & $1.61 \mathrm{~m}$ & \\
\hline IMC & F & 22 & \\
\hline Sexo & M & $65 \%$ & NS \\
& & $60 \%$ & \\
\hline Antecedentes de contacto & $54 \%$ & NS \\
\hline Tratamiento previo & $63 \%$ & NS \\
Resistencia & Rifampicina & $66.70 \%$ & $<0.05$ \\
& Etionamida & $33 \%$ & $<0.05$ \\
& Estreptomicina & $77 \%$ & $<0.05$ \\
\hline
\end{tabular}


etionamida en un $68,1 \%(p<0.05)$ seguido de rifampicina en un $47,2 \%(p<0.05)$ y estreptomicina en un $33 \%(p<0.05)$. Un estudio en Mongolia encontró que la resistencia a la rifampicina se asoció significativamente $(p<0.001)$ con la mutación kat $G$ concluyendo que las cepas resistentes a isoniacida con mutaciones en el gen katG, tenían más probabilidades de haber adquirido resistencia a rifampicina que las cepas con mutaciones en el gen inhA ${ }^{[8]}$, asimismo diferentes estudios revelan que la mutación en el gen katG se asocia con una mayor proporción de resistencia a rifampicina y las mutaciones en el promotor inhA con monorresistencia a isoniacida ${ }^{[9,10]}$.

En conclusión, podemos afirmar que la mutación del gen KatG es la más frecuente en nuestro medio, la cual se asocia en mayor porcentaje con resistencia a estreptomicina seguido de rifampicina y el gen InhA se asocia mayormente con resistencia a etionamida, estos resultados resaltan la importancia de saber que existe resistencia cruzada entre la isoniacida y su respectivo análogo estructural la etionamida, además de resistencia cruzada con la rifampicina y estreptomicina, lo cual nos sugieren valorar la utilidad de etionamida en esquemas de pacientes con TB-MDR con presencia de mutaciones en el gen inhA, asimismo la tuberculosis resistente a isoniacida plantea un desafío para el tratamiento dependiendo de la mutación genética encontrada, permitiendo a los médicos decidir sobre los esquemas terapéuticos en estos pacientes.

Una limitación del estudio es el riesgo de sesgo de selección al incluir una población de casos que se presentaron al CER, por lo que nuestros datos no podrían extrapolarse a la población peruana, sin embargo, es pertinente mencionar que la DIRIS Lima Centro incluye distritos con alta prevalencia de tuberculosis como San Juan de Lurigancho, La Victoria y Cercado de Lima.

Contribuciones de autoría: KVP, JSL, FLLT, CTV Y TSR han participado en la concepción del artículo, recolección de datos, en la redacción y aprobación de la versión final.

Potenciales conflictos de interés: Los autores declaran no tener conflictos de interés.

Fuentes de financiamiento: Autofinanciado.

\section{ORCID}

Kenneth G. Vargas-Ponce ORCID: https://orcid.org/0000-00023233-9426

Claudia L. Trujillo-Valencia ORCID: https://orcid.org/0000-00033707-0662
Trilce Saravia-Ruiz ORCID: https://orcid.org/0000-0002-71075988

Juan A. Salas-López ORCID: https://orcid.org/0000-0003-36866837

Félix K. Llanos-Tejada ORCID: https://orcid.org/0000-0003-18341287

\section{REFERENCIAS BIBLIOGRÁFICAS}

1. OMS. Tuberculosis [Internet]. [citado 2 de julio de 2020]. Disponible en: https://www.who.int/westernpacific/health-topics/ tuberculosis

2. Ministerio de Salud del Perú. Estadística en Salud [Internet]. REUNIS: Repositorio Único Nacional de Información en Salud - Ministerio de Salud. Perú. [citado 2 de junio de 2020]. Disponible en: http:// www.minsa.gob.pe/reunis/index.asp?op=5

3. Alarcón V, Alarcón E, Figueroa C, Mendoza-Ticona A. Tuberculosis en el Perú: Situación epidemiológica, avances y desafíos para su control. Rev Peru Med Exp Salud Pública. 30 de junio de 2017;34(2):299-310

4. Dean AS, Zignol M, Cabibbe AM, Falzon D, Glaziou P, Cirillo DM, et al. Prevalence and genetic profiles of isoniazid resistance in tuberculosis patients: A multicountry analysis of cross-sectional data. PLOS Med. 21 de enero de 2020;17(1):e1003008.

5. Zignol M, Dean AS, Falzon D, van Gemert W, Wright A, van Deun A, et al. Twenty Years of Global Surveillance of Antituberculosis-Drug Resistance. N Engl J Med. 2016 Sep 15;375(11):1081-9.

6. Pitso L, Potgieter S, Van der Spoel van Dijk A. Prevalence of isoniazid resistance-conferring mutations associated with multidrug-resistant tuberculosis in Free State Province, South Africa. South Afr Med J Suid-Afr Tydskr Vir Geneeskd. 28 de agosto de 2019;109(9):659-64.

7. Alagappan C, Sunil Shivekar S, Brammacharry U, Cuppusamy Kapalamurthy VR, Sakkaravarthy A, Subashkumar R, et al. Prevalence of mutations in genes associated with isoniazid resistance in Mycobacterium tuberculosis isolates from re-treated smear-positive pulmonary tuberculosis patients: A meta-analysis. J Glob Antimicrob Resist. 2018;14:253-9.

8. Narmandakh E, Tumenbayar O, Borolzoi T, Erkhembayar B, Boldoo T, Dambaa N, et al. Genetic Mutations Associated with Isoniazid Resistance in Mycobacterium tuberculosis in Mongolia. Antimicrob Agents Chemother Antimicrob Agents Chemother. 2020 Jun 23;64(7):e00537-20.

9. Doorn HR van, Haas PEW de, Kremer K, Vandenbroucke-Grauls CMJE, Borgdorff MW, Soolingen D van. Public health impact of isoniazid-resistant Mycobacterium tuberculosis strains with a mutation at amino-acid position 315 of katG: a decade of experience in The Netherlands. Clin Microbiol Infect. 1 de agosto de 2006;12(8):769-75

10. Hazbón MH, Brimacombe M, Bobadilla del Valle M, Cavatore $M$, Guerrero MI, Varma-Basil M, et al. Population Genetics Study of Isoniazid Resistance Mutations and Evolution of MultidrugResistant Mycobacterium tuberculosis. Antimicrob Agents Chemother. agosto de 2006;50(8):2640-9. 